\title{
Solving the Multi-Commodity Single-Stage Capacitated Warehouse Location Problem Using Bat Algorithm
}

\author{
Abdulgabbar Abdullah Mhana ${ }^{1}$, Hegazy Zaher ${ }^{2}$, Naglaa Ragaa ${ }^{3}$, Heba Sayed ${ }^{4}$ \\ ${ }^{1} \mathrm{PhD}$ Candidate in Operations Research, faculty \\ of graduate studies for statistical research, Cairo \\ University, Egypt \\ ${ }^{2}$ Professor Doctor in Mathematical statistics, \\ faculty of graduate studies for statistical research, \\ Cairo University, Egypt \\ ${ }^{3}$ Associate Professor in Operations Research, \\ faculty of graduate studies for statistical research, \\ Cairo University, Egypt \\ ${ }^{4}$ Doctor in Operations Research, faculty of \\ graduate studies for statistical research, Cairo \\ University, Egypt \\ D.O.I - 10.51201/JUSST/21/05183 \\ http://doi.org/10.51201/JUSST/21/05183
}

\begin{abstract}
The warehouse location problem is so important in supply chains management. In concerns with optimize the assignment of warehouses in order to minimize the total costs related to transportation and service costs. In this paper, a mathematical model for the multi-commodity single-stage capacitated warehouse location problem is presented. A bat algorithm is developed for solving the problem and an illustrative example is presented to show its implementation. The computational results is done on a large sized problem to show the efficiency of the proposed algorithm.
\end{abstract}

\section{Introduction}

The warehouse location problem is one of the problems that appear in supply chains. From the problem name, it can be seen that the problem is concerned with allocating the best warehouses' location to minimize the cost of transportation and warehouse service. The classifications of the warehouse location problems can be described as follows:

- $\quad$ Single stage

- Multi-stage

- Single commodity

- Multi-commodity

- Single period

- Multi-period

- Un-capacitated

- Capacitated

The single-stage warehouse location problem refers to only one stage of warehouses between the plant or the manufacturer and the customer, while the multi-stage warehouse location problem 
refers to more than one stage of warehouses between the plant and the customer. Figure 1 shows the multi-commodity single-stage warehouse location problem [1].

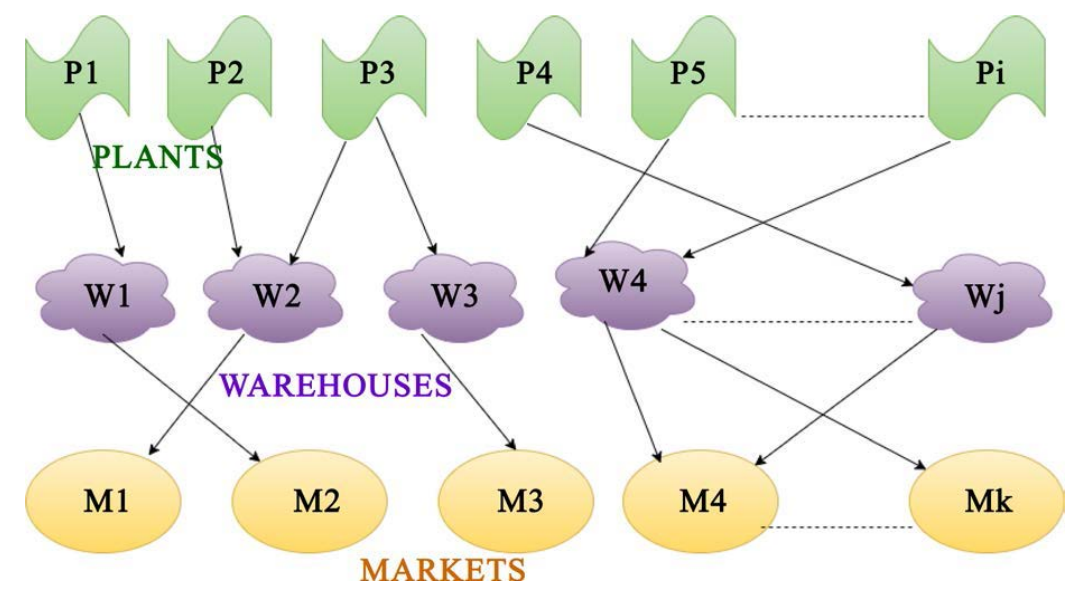

Figure 1: The multi-commodity single-stage warehouse location problem

The selected problem to be solved in this paper is the multi-commodity single-stage capacitated warehouse location problem. The paper is organized as follows. The second section is the literature review. The third section is the mathematical model. The fourth section is the proposed algorithm. The fifth section is illustrative example. The sixth section is a computational result of large sized problem. Eventually, the seventh section is the conclusion.

\section{Literature Review}

Mehrez et al. [2] developed an algorithm that composes the weighted maximin and minmax rectilinear distances for the given demands. Bastian and Volkmer [3] considered the single facility location problem and relocation problem. Wan et al. [4] delineated results of survey on the evaluation of some warehouses location from the flight forwarders in one of the Hong Kong airports. They presented a mathematical model for the warehouse location problem. Chen and Ting [5] developed Lagrangian heuristic and ant colony optimization for solving the single source capacitated facility location problem. Guastaroba and Speranza [6] developed a heuristic for solving the capacitated facility location problem. Contreras and Díaz [7] developed scatter search for solving the capacitated facility location problem. They divide their algorithm into two stages, which are the initialization phase and the improvement phase. They also applied tabu search.

Ho [8] developed an iterated tabu search heuristic for solving the capacitated facility location problem. He used perturbation operators to avoid stuck in local optima. Stanimirovic and Ciric [9] developed an algorithm to solve the single facility min-sum Weber location problem based on the lift metric. Manzour-Al-Ajdad et al. [10] developed a hierarchical heuristic based method for solving the location routing problem. They found the optimal location problem in the continuous plane and determined the paths from the chosen facilities that serve all customers. Pirkul and Jayaraman [11] formulated the multi-commodity facility location problem and solved it using heuristic. Askin et al. [12] considered the problem of designing the distribution network that 
provide products from multiple plants then deliver them to many outlets. They formulated the problem and developed a genetic algorithm for solving the problem. Canel et al. [13] developed a branch and bound with dynamic programming for solving the multi-commodity multi-period multi-stage facility location problem.

\section{The Mathematical Model}

The problem is one of the assignment problems, which can be represented by an integer 0-1 linear programming formulation. Such type of problems is known in research as combinatorial problems which are extremely hard to be solved with classical mathematical approaches. The selected warehouse location problem in this paper is the multi commodity single-stage capacitated warehouse location problem. In such type, the warehouse capacity is known and limited and the capacity of plant for production and warehouse capacity enough to meet the market demands for different commodities. The objective of the problem is to minimize the total cost which includes transportation costs and fixed costs related to establishing the warehouse at a particular location. The formulation of the problem is as follows:

Notation:

$i \quad$ The set of plants (factory) supply point

$j \quad$ The Set of the point warehouse

$k \quad$ The set of the markets

$c w_{j} \quad$ The service cost of warehouse (j).

$C_{i j k} \quad$ The transportation cost from factory i by warehouse $\mathrm{j}$ to market $\mathrm{k}$.

$Q_{i} \quad$ The quantity shipped (transportation) from factory i

$R_{j} \quad$ A very large number, here taken as two times the maximum supply or maximum warehouse capacity.

$S_{k} \quad$ The demand of market $\mathrm{k}$

$\operatorname{Min.} Z=\sum_{i=1}^{l} \sum_{j=1}^{m} \sum_{k=1}^{n} C_{i j k} x_{i j k}+\sum_{i=1}^{l} \sum_{j=1}^{m} \sum_{k=1}^{n} c w_{j} x_{i j k}$

Subject to: 


$$
\begin{aligned}
& \sum_{j=1}^{m} \sum_{k=1}^{n} x_{i j k}=Q_{i}, \forall i \\
& \sum_{i=1}^{l} \sum_{j=1}^{m} x_{i j k}=S_{k}, \forall j \\
& \sum_{i=1}^{l} \sum_{k=1}^{n} x_{i j k} \leq R_{i}, \forall j \\
& x_{i j k} \in\{0,1\}, \forall i, j, k
\end{aligned}
$$

\section{The Proposed Algorithm}

The bat algorithm is inspired from the echolocation of bats. Each bat flies randomly searching for food with a velocity $v_{i}$ and position $x_{i}$ with a diversified frequency $f_{i}$ and loudness $A_{i}$. While searching for food each bat changes its frequency, loudness, and emission rate $r_{i}$, which in turn update velocities and positions. In the algorithm, the random walks of bats, which consists of changing positions and velocities, play the role of exploring diversified solution areas and the loudness rate of each bat plays the role of local search procedure. The emission rate plays the role of deciding to make a local search or not. Zhang et al. [14] shows the steps of the bat algorithm as in algorithm 1 . The velocity and positions of each bat in the algorithm are updated according to equation (6) and (7). The frequency is updated according to equation (8), where the frequency is limited by minimum and maximum value and generated by uniform random number. The loudness rate is a vector that has the same dimension as the position vector for each bat and it is used to apply the local search as in equation (9). Its elements is initialized randomly using uniform distribution. The loudness and emission rate are updated using fixed parameters as seen in equations (10) and (11). In all equations $t$ referes to the iteration number.

$$
\begin{gathered}
v_{i}^{t+1}=v_{i}^{t}+\left(x_{i}^{t}-x^{*}\right) \times f_{i} \\
x_{i}^{t+1}=x_{i}^{t}+v_{i}^{t+1} \\
f_{i}=f_{\text {min }}+\left(f_{\text {max }}+f_{\text {min }}\right) \times \beta \text {, where } \beta \text { is random number } \\
x_{i}^{t+1}=x^{*}+\varepsilon \times \bar{A} \text {, where } \bar{A} \text { is the average loundness } \\
A_{i}^{t+1}=\sigma A_{i}^{t} \\
r_{i}^{t+1}=r_{i}^{0}\left(1-e^{-\gamma t}\right)
\end{gathered}
$$

In the multi-commodity single-stage warehouse location problem, there are three position and velocity vectors for plants, warehouses, and markets. The algorithm 1 will be applied using such three position and velocity vectors. The solution procedure is depending on randomly sorting the plants, warehouses, and markets. That is done using associated vectors that has random numbers in each element of each vector. Then the elements of each vector that has the random numbers are to be sorted in descending order. The output vectors then used to represent the priority of 
plants, warehouses, and markets. Such priorities then are to be used to generate the random bat solution. The mutation procedures afterwards will be done on the associated vectors in order to obtain the local search solutions using loudness rate vectors.

Algorithm 1: The bat algorithm

\section{Begin}

Initialize position, velocity and other parameters of each bat

While (Stop criteria is met?)

Randomly generate the frequency for each bat using equation (8)

Update the velocity using equation (6)

Update the position using equation (6(7)

If $\operatorname{rand}()>r_{i}^{t}$

Update the position using equation (9)

\section{End}

Calculate the fitness

If $\left(\right.$ rand ()$\left.<A_{i}^{t}\right) \& \&\left(f\left(x_{i}^{t}\right)<f\left(x^{*}\right)\right)$

Replace the position with new one

Update the emission rate and loudness using equations (10) and (11)

\section{End}

Update the global best solution by comparing the current global best with the current local best

\section{End}

Output the best global solution

\section{End}

\section{Illustrative Example}

If the problem in hand has 4 plants, 3 warehouses, and 3 markets. Assume that the number of produced products from plant $1,2,3$, and 4 are 10,15, 5, and 20 respectively. The capacity of warehouse 1, 2, and 3 are 15, 15, and 30 products. The required demand from market 1,2 , and 3 are 15, 20, and 25 products. The service cost for warehouses 1,2 , and 3 are 5,7 , and 10 . The transportation costs from plants to warehouses are as in Table 1 . The transportation costs from warehouses and markets are as in Table 2. Then, the associated sorted vectors for the plants, warehouses, and markets can be such as in Table 3, 4, and 5 respectively. The solution using the sorting random vectors is as in Table 6 and 7.

Table 1: The transportation costs from plants to warehouses

\begin{tabular}{|c|c|c|c|}
\cline { 2 - 4 } \multicolumn{1}{c|}{} & Warehouse 1 & Warehouse 2 & Warehouse 3 \\
\hline Plant 1 & 5 & 7 & 3 \\
\hline Plant 2 & 10 & 4 & 8 \\
\hline Plant 3 & 2 & 6 & 4 \\
\hline Plant 4 & 4 & 10 & 5 \\
\hline
\end{tabular}


Table 2: The transportation costs from warehouses to markets

\begin{tabular}{|c|c|c|c|}
\cline { 2 - 4 } \multicolumn{1}{c|}{} & Market 1 & Market 2 & Market 3 \\
\hline Warehouse 1 & 3 & 7 & 10 \\
\hline Warehouse 2 & 5 & 12 & 4 \\
\hline Warehouse 3 & 10 & 2 & 7 \\
\hline
\end{tabular}

Table 3: Sorting the random vector of each plant

\begin{tabular}{|c|c|c|c|c|}
\hline Plants & Plant 4 & Plant 1 & Plant 3 & Plant 2 \\
\hline $\begin{array}{c}\text { Sorted associated } \\
\text { vector }\end{array}$ & 0.94 & 0.75 & 0.33 & 0.25 \\
\hline
\end{tabular}

Table 4: Sorting the random vector of each warehouse

\begin{tabular}{|c|c|c|c|}
\hline Warehouses & Warehouse 2 & Warehouse 1 & Warehouse 3 \\
\hline $\begin{array}{c}\text { Sorted associated } \\
\text { vector }\end{array}$ & 0.88 & 0.64 & 0.11 \\
\hline
\end{tabular}

Table 5: Sorting the random vector of each market

\begin{tabular}{|c|c|c|c|}
\hline Markets & Market 3 & Market 2 & Market 1 \\
\hline $\begin{array}{c}\text { Sorted associated } \\
\text { vector }\end{array}$ & 0.77 & 0.44 & 0.19 \\
\hline
\end{tabular}

Table 6: The solution paths from plants to warehouses

\begin{tabular}{|c|c|}
\hline Plant & Warehouse \\
\hline 4 & 2 \\
\hline 4 & 1 \\
\hline 1 & 1 \\
\hline 3 & 3 \\
\hline 2 & 3 \\
\hline
\end{tabular}

Table 7: The solution paths from warehouses to markets

\begin{tabular}{|c|c|}
\hline Market & Warehouse \\
\hline 3 & 2 \\
\hline 2 & 1 \\
\hline 2 & 3 \\
\hline 1 & 3 \\
\hline
\end{tabular}


The service costs from the obtained by the solution is 22 . The total transportation cost from plants to warehouses is 31. The total transportation cost from warehouses to markets is 23. Then total cost of such random solution is equal to 76 . As seen in the illustrative example, Table 3,4 , and 5 represent the position vectors. Then the positions can be changed using the velocity vectors to have other positions (Solutions). Such process is to be done on the associated vectors. In addition, the local search is to be done using the loudness rate on the associated vectors. For example, Table 8 shows one of the random associated vectors the represent the position of the current bat. In addition, it shows the velocity of the current bat, the position of the best bat in the population of bats, the new position of the current bat after applying the velocity and the frequency that has minimum value equal 0 and maximum value equal 2 .

Table 8: Example of positions and velocities calculations

\begin{tabular}{|c|c|c|c|c|}
\hline $\begin{array}{c}\text { The current } \\
\text { position of } \\
\text { plant's bat }\end{array}$ & 0.75 & 0.25 & 0.33 & 0.94 \\
\hline $\begin{array}{c}\text { The velocity of } \\
\text { the current } \\
\text { plant's bat }\end{array}$ & 0.77 & 0.64 & 0.22 & 0.44 \\
\hline $\begin{array}{c}\text { The best } \\
\text { position of all } \\
\text { plant's bats }\end{array}$ & 0.22 & 0.39 & 0.77 & 0.99 \\
\hline $\begin{array}{c}\text { The new } \\
\text { velocity when } \mathrm{f} \\
=1.5\end{array}$ & 1.57 & 0.43 & -0.44 & 0.37 \\
\hline $\begin{array}{c}\text { The position of } \\
\text { the new bat }\end{array}$ & 2.32 & 0.68 & -0.11 & 1.31 \\
\hline
\end{tabular}

The average loudness will be calculated to be used with the local search equation (9). Then the loudness is to be updated as well as the emission rate using equation (10) and (11). The illustrative example section showed how to construct the solutions using positions. In addition, it shows how to modify the position using velocities. All information related to the algorithm afterward are related to the given parameters of the algorithm.

\section{Computational results}

The proposed bat algorithm is developed using Matlab. In this section, the data a large sized problem is introduced and solved by the proposed algorithm. The data of the problem is as follows: 
Table 9: The transportation costs from plants to warehouses

\begin{tabular}{|c|c|c|c|c|c|c|c|c|}
\hline & \multicolumn{7}{|c|}{ Plants } \\
\hline & & 1 & 2 & 3 & 4 & 5 & 6 & 7 \\
\hline \multirow{6}{*}{ 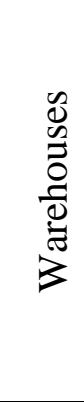 } & 1 & 40 & 38 & 29 & 42 & 67 & 49 & 75 \\
\hline & 2 & 34 & 73 & 87 & 91 & 55 & 80 & 34 \\
\hline & 3 & 80 & 93 & 62 & 97 & 85 & 93 & 83 \\
\hline & 4 & 45 & 67 & 91 & 78 & 30 & 32 & 31 \\
\hline & 5 & 62 & 82 & 55 & 42 & 85 & 76 & 70 \\
\hline & 6 & 62 & 54 & 67 & 48 & 38 & 52 & 95 \\
\hline
\end{tabular}

Table 10: The transportation costs from warehouses to markets of problem 1

\begin{tabular}{|c|c|c|c|c|c|c|c|}
\cline { 3 - 8 } \multicolumn{2}{c|}{} & \multicolumn{7}{c|}{ Warehouses } \\
\cline { 2 - 8 } \multicolumn{2}{c|}{} & 1 & 2 & 3 & 4 & 5 & 6 \\
\hline \multirow{4}{*}{} & 1 & 61 & 62 & 49 & 46 & 76 & 83 \\
\cline { 2 - 8 } & 2 & 27 & 41 & 85 & 39 & 92 & 90 \\
\cline { 2 - 8 } & 3 & 63 & 71 & 68 & 30 & 21 & 73 \\
\cline { 2 - 8 } & 4 & 89 & 98 & 66 & 27 & 38 & 63 \\
\cline { 2 - 8 } & 5 & 40 & 49 & 23 & 92 & 20 & 76 \\
\cline { 2 - 8 } & 6 & 57 & 87 & 39 & 59 & 69 & 22 \\
\cline { 2 - 8 } & 7 & 28 & 57 & 67 & 83 & 45 & 60 \\
\cline { 2 - 8 } & 8 & 80 & 72 & 23 & 86 & 88 & 62 \\
\hline
\end{tabular}

Table 11: The production volume of each plant

\begin{tabular}{|c|c|}
\hline Plant & Production \\
\hline 1 & 72 \\
\hline 2 & 59 \\
\hline 3 & 55 \\
\hline 4 & 39 \\
\hline 5 & 47 \\
\hline 6 & 43 \\
\hline 7 & 25 \\
\hline
\end{tabular}


Table 12: The Capacity and service cost for each warehouse

\begin{tabular}{|c|c|c|}
\hline Warehouse & Capacity & Service Cost \\
\hline 1 & 82 & 25 \\
\hline 2 & 25 & 52 \\
\hline 3 & 71 & 83 \\
\hline 4 & 94 & 60 \\
\hline 5 & 78 & 65 \\
\hline 6 & 50 & 46 \\
\hline
\end{tabular}

Table 13: The demand of each market

\begin{tabular}{|c|c|}
\hline Market & Demand \\
\hline 1 & 27 \\
\hline 2 & 62 \\
\hline 3 & 59 \\
\hline 4 & 49 \\
\hline 5 & 22 \\
\hline 6 & 24 \\
\hline 7 & 61 \\
\hline 8 & 36 \\
\hline
\end{tabular}

The parameters of the proposed bat algorithm is tuned empirically to find the best parameter levels. It found that the best parameter levels for each parameter is 100 bat per population, the minimum and maximum frequency are 0 and 2, the number of iterations is 20, alpha and gamma parameters that are related to loudness and emission update are equal to 0.5 and 0.4 . After solving the problem using the tuned parameters, the obtained solution has total cost equal to 1384. The solution is as follows:

Table 14: The selected warehouses to transport products from plants

\begin{tabular}{|c|c|c|c|c|c|c|c|}
\hline \multicolumn{7}{c|}{} & \multicolumn{7}{c|}{ Warehouses } \\
\hline \multirow{4}{*}{ 売 } & 0 & 0 & 1 & 1 & 1 & 0 & 0 \\
\cline { 2 - 8 }$\sim$ & 0 & 0 & 0 & 0 & 0 & 0 & 0 \\
\cline { 2 - 8 } & 1 & 0 & 0 & 0 & 0 & 0 & 0 \\
\cline { 2 - 8 } & 1 & 0 & 0 & 0 & 1 & 1 & 1 \\
\cline { 2 - 8 } & 0 & 1 & 0 & 0 & 0 & 0 & 0 \\
\cline { 2 - 8 } & 0 & 1 & 1 & 0 & 0 & 0 & 0 \\
\hline
\end{tabular}


Table 15: the selected warehouses to transport products to markets

\begin{tabular}{|c|c|c|c|c|c|c|}
\hline & \multicolumn{6}{|c|}{ Warehouses } \\
\hline \multirow{5}{*}{ 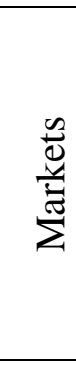 } & 0 & 0 & 0 & 0 & 1 & 0 \\
\hline & 1 & 0 & 0 & 1 & 0 & 0 \\
\hline & 0 & 0 & 1 & 1 & 0 & 0 \\
\hline & 0 & 0 & 0 & 0 & 1 & 1 \\
\hline & 0 & 0 & 1 & 0 & 0 & 0 \\
\hline & 1 & 0 & 0 & 0 & 0 & 1 \\
\hline & 1 & 0 & 0 & 0 & 0 & 0 \\
\hline & 0 & 0 & 1 & 0 & 0 & 0 \\
\hline
\end{tabular}

In Tables 14 and 15, the ones refer to the selected warehouses and the zeros refer to the unselected warehouses.

\section{Conclusion}

This paper introduced a mathematical model for the multi-commodity single-stage warehouse location problem. A bat algorithm is developed for solving such problem. An illustrative example is presented to show the implementation of how to represent the position vectors and velocities. In the computational result, a large sized problem, which has 7 warehouses, 6 plants, and 8 markets, is presented after coding the bat algorithm using Matlab and tuning the parameters of the algorithm empirically. After solving the problem, it found that best result of the algorithm is that the total cost is equal to 1384 .

\section{References}

[1] R. R. K. Sharma, A. Malviya, V. Kumar, V. Singh, and P. Agarwal, "Application of Modified Benders Decomposition to Single-Stage Multi-Commodity Multi-Period Warehouse Location Problem: An Empirical Investigation,” Am. J. Oper. Res., vol. 06, no. 03, pp. 245-259, May 2016, doi: 10.4236/ajor.2016.63025.

[2] A. Mehrez, Z. Sinuany-Stern, and A. Stulman, "A single facility location problem with a weighted maximin-minimax rectilinear distance,” Comput. Oper. Res., vol. 12, no. 1, pp. 51-60, Jan. 1985, doi: 10.1016/0305-0548(85)90006-1.

[3] M. Bastian and M. Volkmer, "A perfect forward procedure for a single facility dynamic location/relocation problem,” Oper. Res. Lett., vol. 12, no. 1, pp. 11-16, Jul. 1992, doi: 10.1016/0167-6377(92)90016-V.

[4] Y. W. Wan, R. K. Cheung, J. Liu, and J. H. Tong, "Warehouse location problems for air freight forwarders: A challenge created by the airport relocation,” J. Air Transp. Manag., vol. 4, no. 4, pp. 201-207, Oct. 1998, doi: 10.1016/S0969-6997(98)00024-6.

[5] C. H. Chen and C. J. Ting, "Combining Lagrangian heuristic and Ant Colony System to 
solve the Single Source Capacitated Facility Location Problem," Transp. Res. Part E Logist. Transp. Rev., vol. 44, no. 6, pp. 1099-1122, Nov. 2008, doi: 10.1016/j.tre.2007.09.001.

[6] G. Guastaroba and M. G. Speranza, "A heuristic for BILP problems: The Single Source Capacitated Facility Location Problem,” Eur. J. Oper. Res., vol. 238, no. 2, pp. 438-450, Oct. 2014, doi: 10.1016/j.ejor.2014.04.007.

[7] I. A. Contreras and J. A. Díaz, "Scatter search for the single source capacitated facility location problem,” Ann. Oper. Res., vol. 157, no. 1, pp. 73-89, Jan. 2008, doi: 10.1007/s10479-007-0193-1.

[8] S. C. Ho, "An iterated tabu search heuristic for the single source capacitated facility location problem," Appl. Soft Comput. J., vol. 27, pp. 169-178, Feb. 2015, doi: 10.1016/j.asoc.2014.11.004.

[9] P. S. Stanimirovic and M. Ciric, "Single-facility Weber Location Problem based on the Lift Metric,” May 2011, Accessed: May 22, 2021. [Online]. Available: http://arxiv.org/abs/1105.0757.

[10] S. M. H. Manzour-Al-Ajdad, S. A. Torabi, and S. Salhi, "A hierarchical algorithm for the planar single-facility location routing problem,” Comput. Oper. Res., vol. 39, no. 2, pp. 461-470, Feb. 2012, doi: 10.1016/j.cor.2011.05.013.

[11] H. Pirkul and V. Jayaraman, "A multi-commodity, multi-plant, capacitated facility location problem: Formulation and efficient heuristic solution," Comput. Oper. Res., vol. 25, no. 10, pp. 869-878, Oct. 1998, doi: 10.1016/S0305-0548(97)00096-8.

[12] R. G. Askin, I. Baffo, and M. Xia, "Multi-commodity warehouse location and distribution planning with inventory consideration,” Int. J. Prod. Res., vol. 52, no. 7, pp. 1897-1910, 2014, doi: 10.1080/00207543.2013.787171.

[13] C. Canel, B. M. Khumawala, J. Law, and A. Loh, "An algorithm for the capacitated, multi-commodity multi-period facility location problem,” Comput. Oper. Res., vol. 28, no. 5, pp. 411-427, Apr. 2001, doi: 10.1016/S0305-0548(99)00126-4.

[14] M. Zhang, Z. Cui, Y. Chang, Y. Ren, X. Cai, and H. Wang, "Bat algorithm with individual local search," in IFIP Advances in Information and Communication Technology, Nov. 2018, vol. 539, pp. 442-451, doi: 10.1007/978-3-030-01313-4_47. 